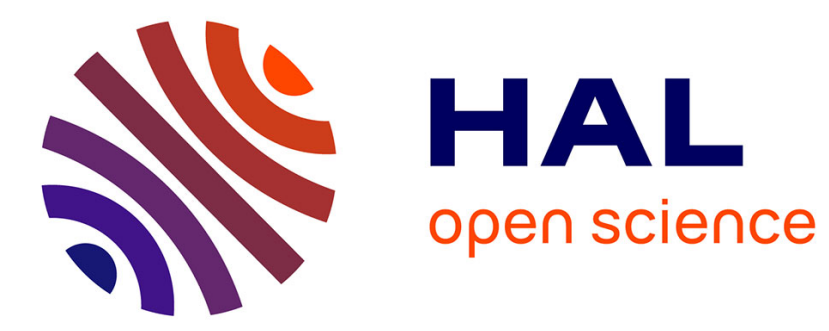

\title{
GABAergic hub neurons orchestrate synchrony in developing hippocampal networks.
}

Paolo Bonifazi, Miri Goldin, Michel A. Picardo, Isabel Jorquera, A. Cattani, Gregory Bianconi, Alfonso Represa, Yehezkel Ben-Ari, Rosa Cossart

\section{- To cite this version:}

Paolo Bonifazi, Miri Goldin, Michel A. Picardo, Isabel Jorquera, A. Cattani, et al.. GABAergic hub neurons orchestrate synchrony in developing hippocampal networks.. Science, 2009, 326 (5958), pp.1419-24. 10.1126/science.1175509 . inserm-00483216

\section{HAL Id: inserm-00483216 https://www.hal.inserm.fr/inserm-00483216}

Submitted on 12 May 2010

HAL is a multi-disciplinary open access archive for the deposit and dissemination of scientific research documents, whether they are published or not. The documents may come from teaching and research institutions in France or abroad, or from public or private research centers.
L'archive ouverte pluridisciplinaire HAL, est destinée au dépôt et à la diffusion de documents scientifiques de niveau recherche, publiés ou non, émanant des établissements d'enseignement et de recherche français ou étrangers, des laboratoires publics ou privés. 
P. Bonifazi, et al.

Science 326, 1419 (2009);

DOI: $10.1126 /$ science. 1175509

The following resources related to this article are available online at www.sciencemag.org (this information is current as of December 4, 2009 ):

Updated information and services, including high-resolution figures, can be found in the online version of this article at:

http://www.sciencemag.org/cgi/content/full/326/5958/1419

Supporting Online Material can be found at:

http://www.sciencemag.org/cgi/content/full/326/5958/1419/DC1

This article cites $\mathbf{4 3}$ articles, 20 of which can be accessed for free:

http://www.sciencemag.org/cgi/content/full/326/5958/1419\#otherarticles

This article appears in the following subject collections:

Neuroscience

http://www.sciencemag.org/cgi/collection/neuroscience

Information about obtaining reprints of this article or about obtaining permission to reproduce this article in whole or in part can be found at:

http://www.sciencemag.org/about/permissions.dtl 


\section{GABAergic Hub Neurons Orchestrate Synchrony in Developing Hippocampal Networks}

\author{
P. Bonifazi, ${ }^{1 *}$ M. Goldin, ${ }^{1 *}$ M. A. Picardo, ${ }^{1}$ I. Jorquera $^{1}{ }^{1}$ A. Cattani, ${ }^{1}$ \\ G. Bianconi, ${ }^{2}$ A. Represa, ${ }^{1}$ Y. Ben-Ari, ${ }^{1}$ R. Cossart ${ }^{1} \dagger$
}

Brain function operates through the coordinated activation of neuronal assemblies. Graph theory predicts that scale-free topologies, which include "hubs" (superconnected nodes), are an effective design to orchestrate synchronization. Whether hubs are present in neuronal assemblies and coordinate network activity remains unknown. Using network dynamics imaging, online reconstruction of functional connectivity, and targeted whole-cell recordings in rats and mice, we found that developing hippocampal networks follow a scale-free topology, and we demonstrated the existence of functional hubs. Perturbation of a single hub influenced the entire network dynamics. Morphophysiological analysis revealed that hub cells are a subpopulation of $\gamma$-aminobutyric acid-releasing (GABAergic) interneurons possessing widespread axonal arborizations. These findings establish a central role for GABAergic interneurons in shaping developing networks and help provide a conceptual framework for studying neuronal synchrony.

$\mathrm{T}$ he coordinated activation of neuronal assemblies features in most physiological brain functions and influences proper network wiring during development (1-3). In addition to cellular excitability, synaptic efficacy, and the balance of excitation and inhibition, the architec-

Fig. 1. Analysis of multineuron calcium activity reveals a scale-free topology in the developing hippocampus. (A) (1) Two-photon calcium fluorescence image of a rat hippocampal slice loaded with Fura$2 A M$ and visualized with multibeam excitation at $\times 10$ magnification. Scale bar, $100 \mu \mathrm{m}$. DG, dentate gyrus. (2) Detected contours of the cells from the fluorescence image shown in (1). Red dots are the 10 highest-connectivity neurons in the represented network based on the analysis of calcium event onsets; gray lines mark the output links of one HC neuron. (3) Probability distribution plot of the fraction of output links over the total population of active neurons imaged with $\times 10$ magnification (gray line, $n=3224$ neurons in four slices; SOM). The graph is plotted on a loglog scale, and a power-law distribution with a slope $(\gamma)$ of $1.3 \pm 0.1(n=4)$ is indicated in black (SOM). The inset shows the location of the 30 highest-connectivity neurons ( $1 \%$ of the population, red dots) on a schematic representation of the hippocampus. The dashed rectangle indicates the size of the area of a $\times 20$ movie. Scale bar, $100 \mu \mathrm{m}$. (B) (1) Same as (A1) but at x20 magnification. The imaged region corresponds to $C A 3 b / c$; that is, around the dotted area in (A1). Arrow indicates the direction of the dentate gyrus. Scale bar, $100 \mu \mathrm{m}$. (2) Same as (A2) but for the movie taken in B1. (3) Same as (A3) but for a population of 7588 neurons. The probability distribution of output links from smaller CA3 regions also follows a power law with a similar scaling power $(\gamma=1.1 \pm 0.1, n=$ 45). The probability threshold for $\mathrm{HC}$ neurons was fixed to $40 \%$ (red-shaded area). The inset indicates the location of HC neurons (red dots) on a schematic representation of the $\mathrm{CA3b} / \mathrm{c}$ region of the hippocampus. HC neurons represented $5 \pm 1 \%(n=45)$ of all functionally connected cells. This value was not significantly different from that calculated in appealing models for brain connectivity because they offer a compromise between computational needs, wiring economy, and robustness $(1,10-14)$. These complex topologies have been found in contexts as diverse as the Internet, social sciences, or biology $(8,15)$. When applied to neuronal circuits, both models share one common feature: Although most neurons are connected locally, a few "hub" neurons possess long-range connections that link large numbers of cells, thereby bestowing network-wide synchronicity. It has been proposed that neuronal hubs orchestrate behaviorally relevant activity in cortical assemblies, as well as being causal in producing pathological oscillations $(4-6,16)$. However, the existence of neuronal hubs is still speculative, perhaps because of the conceptual and technical difficulties of investigating them, including the rarity of high-connectivity (HC) as compared to low-connectivity (LC) cells. Additionally, definitive functional confirmation that neuronal hubs play a key role in synchroni-

${ }^{1}$ Institut de Neurobiologie de la Méditerranée, INSERM U901, Université de la Méditerranée, Parc Scientifique de Luminy, Boîte Postale 13, 13273 Marseille Cedex 9, France. Department of Physics, Northeastern University, Boston, MA 02115 , USA.

*These authors contributed equally to this work.

†To whom correspondence should be addressed. E-mail: cossart@inmed.univ-mrs.fr
A

1

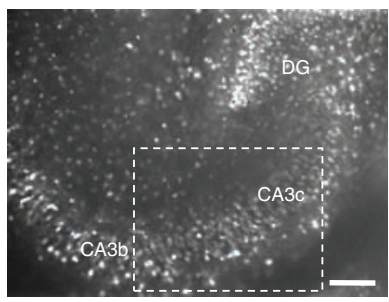

2

3
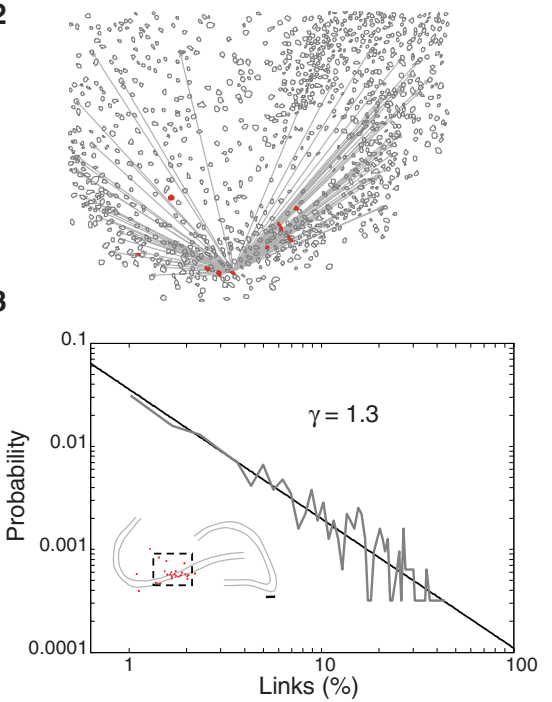

B
1

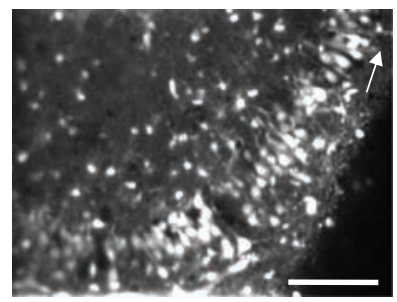

2

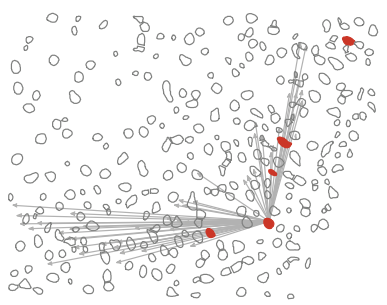

3

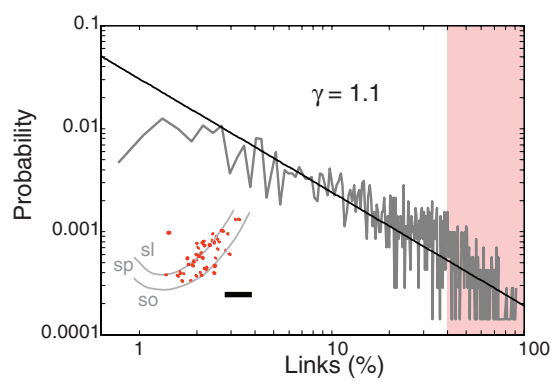

subfields from $\times 10$ data sets of the same size as $\times 20$ images, because $\mathrm{HC}$ neurons represented $4 \pm 1 \%$ of the connected cell population in $\times 10$ movies $(n=4, P>$ 0.05 , Student's $t$ test). sl, stratum lucidum; sp, stratum pyramidale; so, stratum oriens. Scale bar, $100 \mu \mathrm{m}$. 
zation processes requires testing the causal influence of HC cells on network dynamics, something that cannot be achieved with post hoc data analysis $(13,17,18)$.

To find cells involved in the synchronization of neuronal networks, we designed a method to map functional connectivity (FC) in real time in living brain slices, based on the analysis of multineuron calcium activity. Here we use the term FC to denote the statistical relationship between the activities of neurons (19), which should not be confused with the effective connectivity of functional synapses $(20,21)$. This enabled us to perform targeted electrophysiological recordings and stimulation of neurons with a known degree of FC, while imaging network dynamics. We analyzed the developing hippocampal network because it provides an ideal circuit in which to investigate the existence of hub cells. First, as in most developing brain structures, network activity is concentrated in rhythmic synapse-driven synchronizations, the giant depolarizing potentials (GDPs) (3, 22). Second, the network topology underlying the generation of GDPs is confined to local CA3 circuits in slices (23-25), which substantially simplifies the experimental approach. Last, understanding the cellular basis of synchronization in developing circuits is important, because several maturation processes rely on early network oscillations (22).

Using multibeam two-photon excitation of hippocampal slices from rats and GAD67-green fluorescent protein (GFP) knockin (KI) mice [5 to 7 days old; see the supporting online material (SOM)] loaded with the calcium indicator Fura-2AM (26), spontaneous multineuron activity was recorded with a temporal resolution of 50 to $150 \mathrm{~ms}$ (Fig. 1 and fig. S1). The FC of the hippocampus was first investigated at a large scale (with a $\times 10$ objective, Fig. 1A). Focusing on the CA3 region, the activity of $806 \pm 155$ cells ( $n=4$ slices), distributed across the dentate gyrus to the CA1 region, were simultaneously imaged. Focusing on temporal correlations, a functional connection directed from neuron A to neuron $B$ was established if the activation of A consistently preceded that of B (SOM and fig. S1B). An FC map was thus constructed for all recorded neurons (Fig. 1). In all slices imaged at low magnification, the average distribution of the number of output links per neuron was best fitted by a power-law function with an average scaling power of $-1.3 \pm 0.1$ ( $n=4$ slices, Fig. 1 and SOM). Power-law distributed connectivity is the signature of a scale-free topology, in which hubs are rare neurons with a high connectivity index (8). Neurons with the highest connectivity tended to concentrate more often in the CA3c region (Fig. 1A3, inset). Previous studies have reported that this particular area is a preferential site of initiation for spontaneous GDPs $(25,27)$.

To increase the chances of finding hub neurons, we next performed experiments in the CA3c area at higher magnification (with a $\times 20$ objective, Fig. 1B). As previously reported (26), we were able to combine targeted electrophysiological recordings with calcium imaging. Out of 142 neurons recorded while imaging, only 45 were included in the following analysis because estimation and probing of network topology required very stable experimental conditions (SOM). The connectivity of the networks imaged at x 20 was also distributed as a power law with an average scaling factor of $-1.1 \pm 0.1(n=45$ slices; Fig. 1 and SOM). HC neurons were preferentially located in the stratum oriens and lucidum at the

A

LC Neuron

B

1
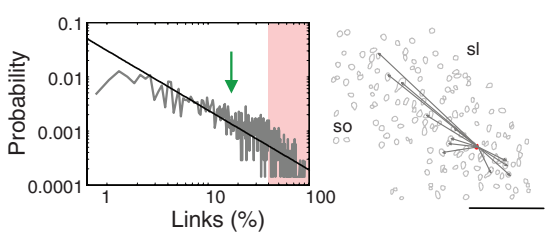

1

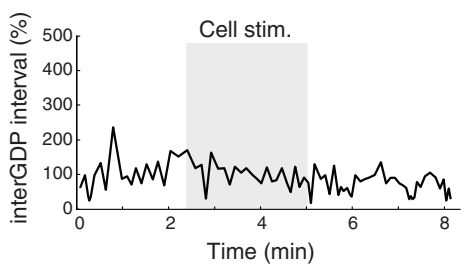

3

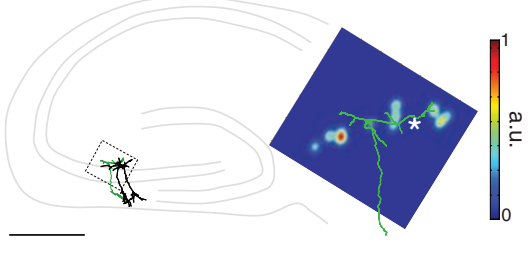

3

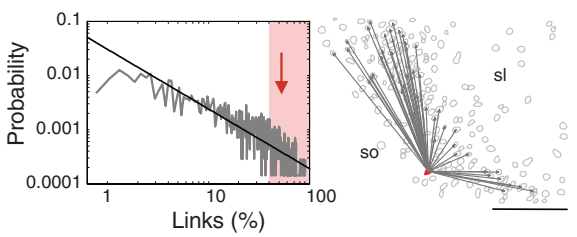

2
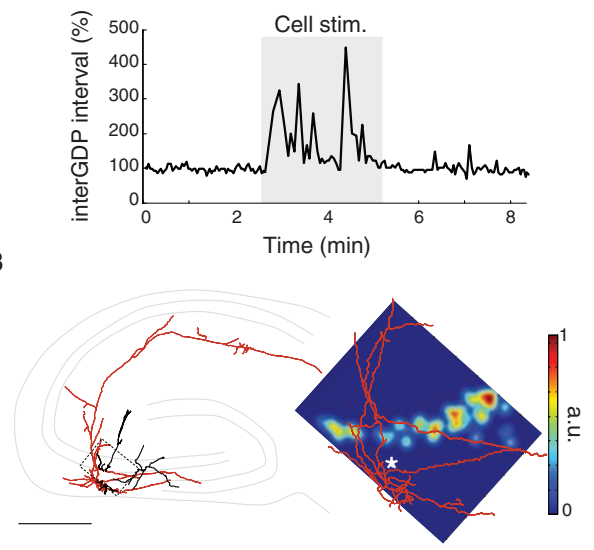

1
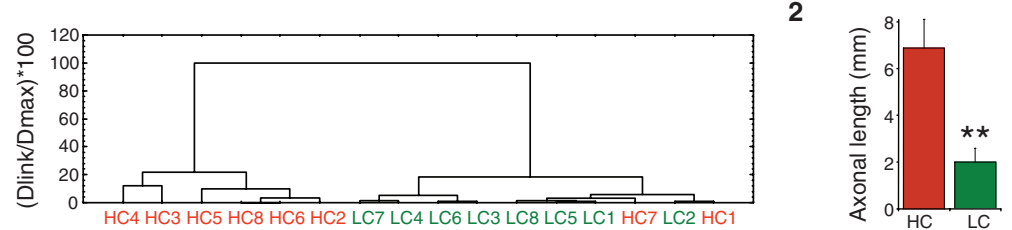

Fig. 2. Stimulation of HC but not LC neurons affects network dynamics. (A) Data from a representative LC interneuron. (1) The green arrow indicates the position in the pooled power-law distribution of output links (Fig. 1B) of the recorded neuron. Red-shaded area indicates the HC region, considering a $40 \%$ probability threshold. The right contour plot shows the position (solid red dot) and output connections (gray lines) of the illustrated LC interneuron. sl, stratum lucidum; so, stratum oriens. Scale bar, $100 \mu \mathrm{m}$. (2) Phasic currentclamp stimulation (200-ms pulses of 75-pA current every $10 \mathrm{~s}$, gray area) of the LC interneuron while being imaged did not affect the occurrence of GDPs (detected from the calcium activity). The interval between GDPs as a function of time is plotted. Values are expressed relative to the average interval between GDPs calculated before the stimulation period. (3) Neurolucida reconstruction of the recorded cell on a schematic representation of the hippocampus reveals an interneuron-like morphology displaying a local axonal arborization (green). Dendritic arborization is black. The black rectangle marks the imaged region. Scale bar, $500 \mu \mathrm{m}$. This is a color-coded representation of the functional connectivity map [same as (A1)] (SOM) overlaid with the axonal morphology (green) of the cell. The asterisk indicates the cell body position. Red represents high cell density (a.u., arbitrary units). (B) Same as (A) but for a representative HC interneuron. Phasic stimulation of the HC interneuron [same protocol as (A2)] significantly decreased GDP frequency [(2), $P<0.05]$. The recorded cell displayed a widespread axonal arborization (red) spanning locally toward the cells functionally connected [(3), right panel] and further toward the dentate gyrus and the CA1 region [(3), left panel]. (C) (1) Cluster analysis tree of the morphological variables describing the 16 recorded and imaged interneurons (Ward's method, Dlink: Euclidian distances, see SOM). Distances were normalized. Most $\mathrm{HC}$ and LC interneurons (based on the analysis of the imaging data) segregated in two different groups. (2) The total axonal lengths of $\mathrm{HC}$ and $\mathrm{LC}$ interneurons were statistically different $(P<0.01)$. 
repeated at 0.1 to $0.2 \mathrm{~Hz}$ (the frequency range of GDPs occurrence)]; and (ii) tonic stimulation (continuous positive or negative current injections, bringing the cell to a membrane potential where it fired continuously or was completely silenced, respectively). Cell/network interaction was estimated using three metrics (SOM): (i) the frequency
A

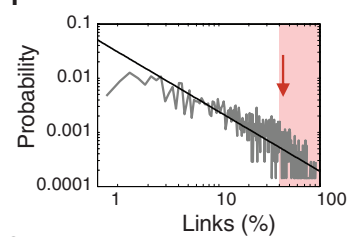

2
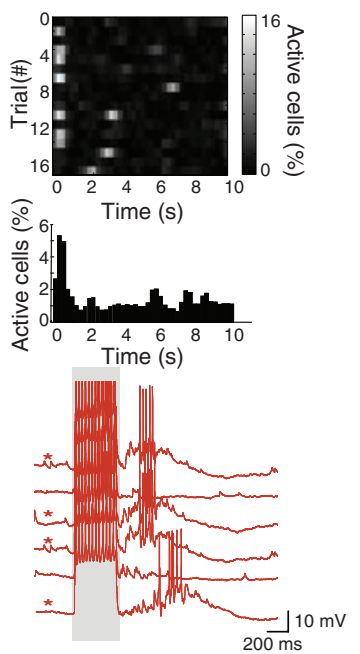

3

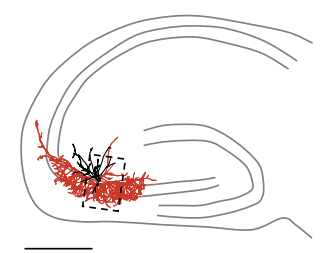

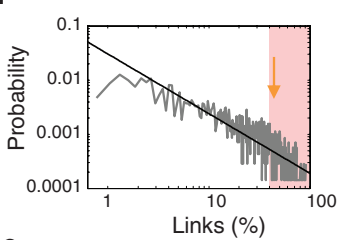

2
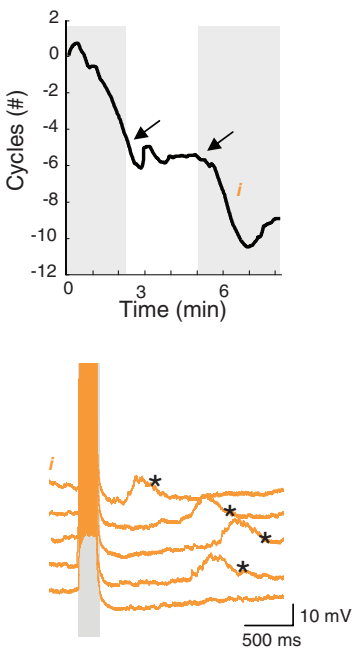

3

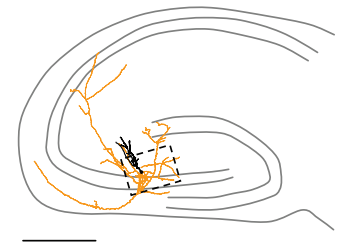

C

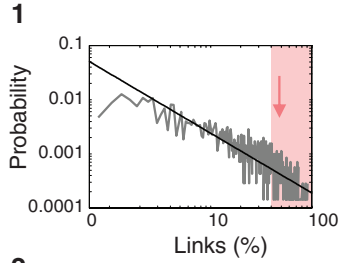

2
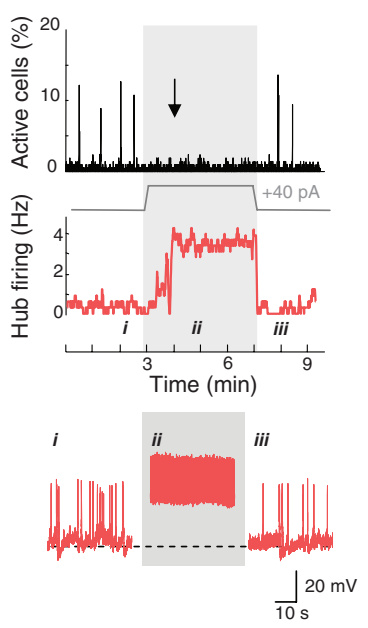

3

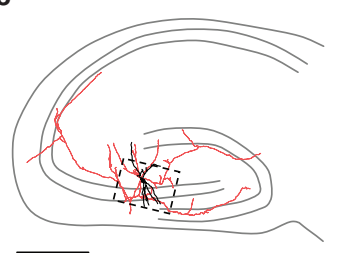

Fig. 3. Perturbations of network dynamics induced by the stimulation of HC interneurons. (A) Data obtained from a $\mathrm{HC}$ interneuron triggering network synchrony $(P<0.05)$. Frame rate, $10 \mathrm{~Hz}$. (1) The red arrow indicates the position in the pooled power-law distribution of output links (Fig. 1B) of the recorded neuron. (2) Fraction of cells active as a function of time after repetitive phasic stimulation (200-ms pulses of $100-\mathrm{pA}$ current every $10 \mathrm{~s}$ ) of the $\mathrm{HC}$ interneuron (16 consecutive trials). The peristimulus time histogram shows the average across different trials. Red traces are current-clamp recordings from the stimulated $\mathrm{HC}$ neuron for six consecutive stimulations (gray). Four out of six trials (indicated by red asterisks in lower panel) triggered GDPs appearing as polysynaptic membrane potential depolarizations. (3) Neurolucida reconstruction of the recorded HC cell on a schematic drawing of the hippocampus. Axonal arborization is in color; dendrites are black. The dashed rectangle indicates the imaged region. Scale bar, $500 \mu \mathrm{m}$. (B) Same as (A) but for a HC interneuron inducing a phase succession of GDPs when stimulated $(P<0.05)$. Phase succession is illustrated in the top graph of $(2)$ plotting the number of GDP cycles skipped during phasic stimulation (gray) as a function of time. The number of expected GDPs was calculated during resting conditions (white) based on the average interval between GDPs. Arrows indicate transitions between oscillatory regimes. Current-clamp recordings from five consecutive stimulation trials for the period marked by (i) show the progressive delay in the occurrence of a GDP (black asterisks) after stimulation (gray). (C) Same as (A) but in a HC interneuron preventing GDPs when stimulated. Graphs in (2) show the fraction of active cells (top histogram), as well as the cell firing frequency (middle), as a function of time. Peaks of synchronous activity (GDPs) disappear when the membrane potential of the cell (bottom) is depolarized by continuous positive current injection ( $40 \mathrm{pA}$; SOM). Current-clamp traces show the activity in the $\mathrm{HC}$ neuron in resting ( $\mathrm{i}$ and iii) and stimulated (ii, gray) conditions. The black arrow indicates the time when a significant effect on network dynamics starts $(P<0.05)$. this way, the number of observed versus expected GDPs was estimated over time (SOM). A cell was considered as affecting network dynamics significantly if it satisfied any of the above criteria.

About a third ( 8 out of 20 neurons) of the targeted HC cells exhibited a significant cell/ network interaction (Figs. 2B and 3). In contrast, no LC neuron but one showed any significant cell/network interaction ( 24 out of 25 neurons; Fig. 2A and fig. S2A). The effects of neurons significantly affecting network dynamics $(n=9)$ could be summarized as follows (Fig. 3): (i) in four cases, tonic or phasic stimulation induced sustained action potential (AP) firing that significantly decreased the occurrence of GDPs to $48 \pm$ $13 \%$ of resting conditions $(P<0.05$; Figs. $2 \mathrm{~B}$ and $3 \mathrm{C}$ and movie $\mathrm{S} 1$ ); (ii) in three cells, phasic stimulation triggered network synchrony in the form of GDPs in $37 \pm 4 \%$ of the trials within $1 \mathrm{~s}$ after the stimulus $(P<0.05$; Fig. 3A and fig. S5); (iii) in three cells, phasic stimulations induced a phase succession of GDPs as compared to resting conditions (Fig. 3B, $P<0.05$ ). Our evidence suggests that these neurons may act like functional hubs. We will henceforth refer to these as hub neurons.

The developing hippocampal network comprises two major cell types: pyramidal glutamatergic cells and $\gamma$-aminobutyric acid-releasing (GABAergic) interneurons. In adult cortical structures, network function is strongly modulated by the action of GABAergic interneurons that represent a minority of the total population but include a variety of subtypes (28). Half of the experiments were performed in GAD67-GFP KI mice (29) to selectively identify GABAergic neurons. All hub neurons recorded in GAD67GFP KI mice, based on their HC index, were GFP-positive (fig. S3, $n=4$ ). Accordingly, the fraction of GFP-positive cells was four times higher in the $\mathrm{HC}$ region than in the total cell population $(22 \%$ of $\mathrm{HC}$ neurons versus $6 \%$ of all neurons, $n=46$ movies in GAD67-GFP KI mice). Therefore, hub neurons are GABAergic, and we next examined whether they represented a specific morphological population. While being recorded, cells were filled with biocytin. All nine hub cells were aspiny neurons and often possessed multipolar dendrites and a cell body located at the border between the pyramidal cell layer and the stratum oriens or lucidum. All HC neurons that were not hubs were morphologically identified as pyramidal cells (fig. S2; 4 cells reconstructed). LC cells not influencing network dynamics exhibited either interneuronal or pyramidal cell morphology (Fig. 2 and fig. S2). All hub neurons had distinctive morphological features, displaying a widespread axonal arborization that most often crossed subfield boundaries, running parallel to principal cell layers toward both the dentate gyrus and CA1 region ( $n=6$ of 9 neurons, Figs. 2 and 3). Three of the hub cells exhibited dense preferential innervation of the CA3 principal cell layer, suggesting a perisomatic, basketlike (28) interneuron subtype (Fig. 3A and fig. S3B2). We 
A
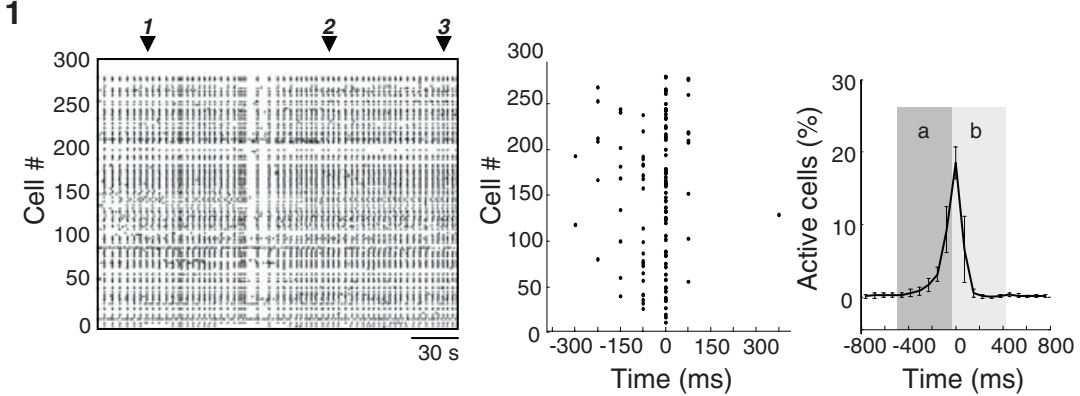

2
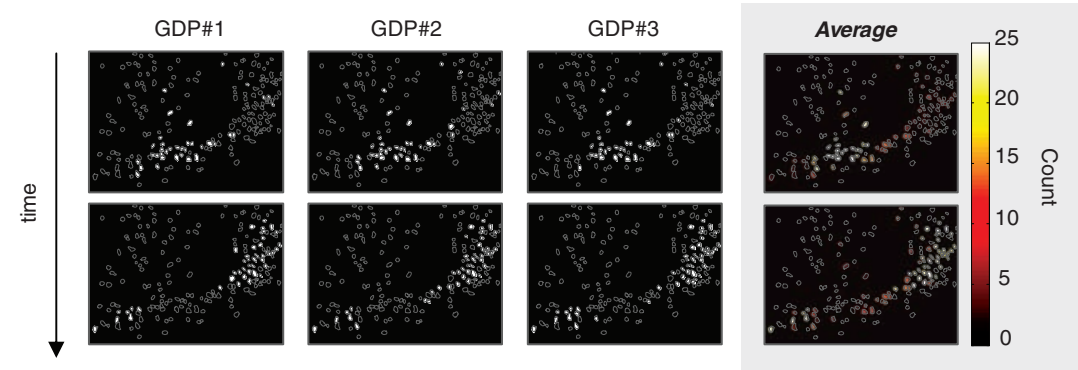

B

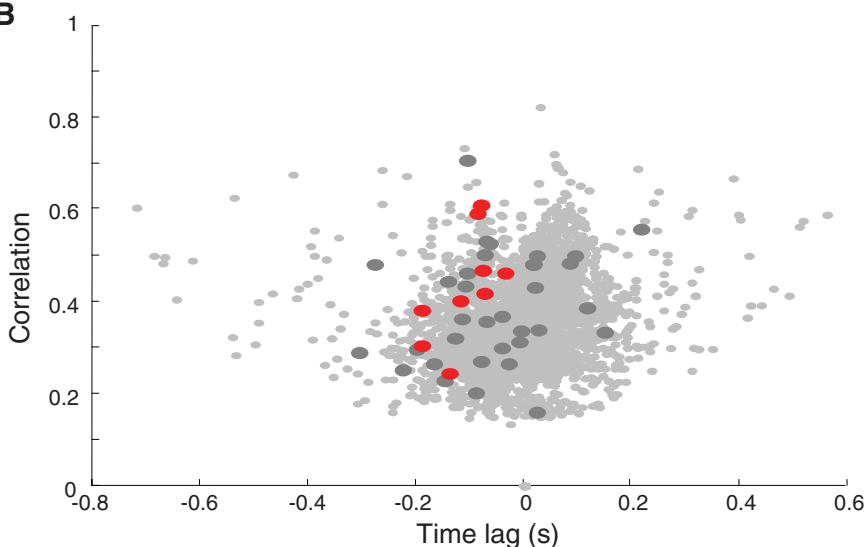

C
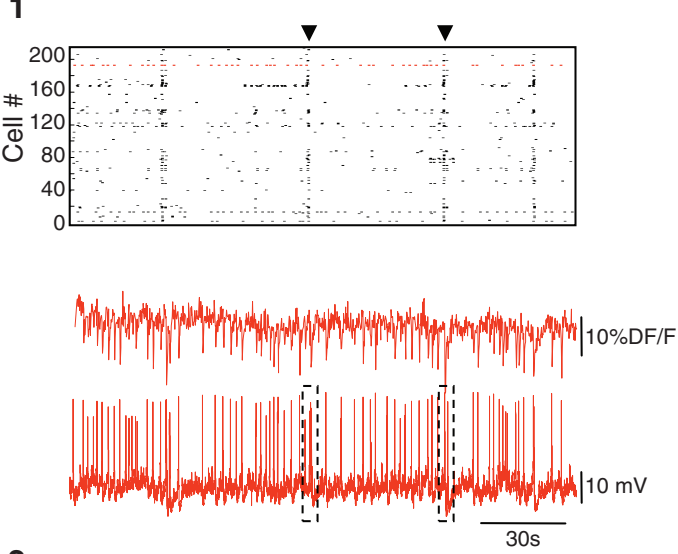

2
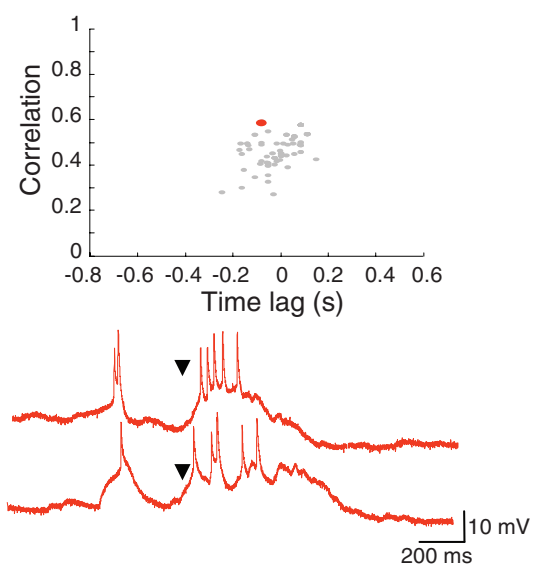

Fig. 4. Hub neurons are activated at the onset of spontaneous network synchronizations. (A) (1) Raster plot of the onsets of calcium events in a representative movie (frame rate, $20 \mathrm{~Hz}$ ). There are many spontaneous GDPs appearing as broken vertical lines in the raster plot. The middle raster plot shows GDP1 on an expanded time scale. The right plot represents the average temporal profile of the fraction of cells sequentially activated in all GDPs recorded in the same network. The peak of cell coactivation was used as zero time reference. Error bars indicate SDs. (2) Contour plots showing cells activated during the buildup [region a in (1)] and at the peak (b) for the three GDPs marked by arrows in the raster plot in (1). Note the similarity between the patterns of cells. Color-coded contour plots on the right quantify how many times a cell is recruited in one of the two temporal windows over 25 representative GDPs. (B) Time-correlation graph (SOM), plotting for each imaged neuron (7588 neurons) the average correlation and average time of activation relative to all other cells. Red dots indicate targeted functional hubs and dark gray dots indicate all other recorded neurons. (C) (1) Raster plot [calculated as in (A1)] of the network activity (frame rate, $6.67 \mathrm{~Hz}$ ) while recording an $\mathrm{HC}$ hub neuron (red dots) in current-clamp mode (bottom trace). Calcium events (top trace) reflect spiking activity (bottom trace). (2) Same timecorrelation graph as in (B) but only for the recorded slice shown in (C1). The red dot marks the HC hub neuron. Red traces show current-clamp recordings of spontaneous activity in the hub neuron at the time of two different GDPs [marked by arrowheads; dashed rectangles in (C1), lower panel]. AP firing occurs in the hub neuron about 200 ms before GDPs. next performed a multivariate analysis of the morphometric data of eight $\mathrm{HC}$ and eight LC interneurons (SOM). Hub interneurons significantly differed from LC interneurons by the length of their axonal tree $(6865 \pm 1238 \mu \mathrm{m}$ versus $2150 \pm 483 \mu \mathrm{m}, P<0.01, n=16$ cells, Fig. 2 ). Given their extended morphology, it seems probable that hub neurons have a higher probability of being severed in brain slices than other cells and thus probably represent a higher fraction of neurons in vivo [but see (20)]. We conclude that functional hubs are GABAergic interneurons with a long axonal arborization. Hub neurons therefore have the features required to activate many postsynaptic targets.

In order to determine the nature of the functional link between hub neurons and other cells, we first asked whether stimulation of hub neurons could directly trigger a calcium response in other neurons, even in the case where the net effect of stimulation was to desynchronize activity. We thus compared functional and effective connectivity maps (SOM and fig. S4). We found that there was a large overlap between the two maps in the case of $\mathrm{HC}$ interneurons $(53 \pm 6 \%$ on average, $n=5$ ), whereas stimulation of $\mathrm{HC}$ pyramids activated only $8 \pm 1 \%$ of functionally connected neurons $(n=5)$, indicating that these were effectively not connected to follower cells. We next performed targeted paired recordings from $\mathrm{HC}$ and follower neurons ( $n=16$ pairs). In the case of $\mathrm{HC}$ interneurons, we observed a $37 \%$ probability of finding a monosynaptic GABAergic connection between neurons ( $n=8$ pairs, fig. S5). This was significantly different from the case of HC pyramidal cells, because no direct connection could be revealed when recording from them ( $n=8$ pairs, $P<0.05$, Wilcoxon-Mann-Whitney two-sample rank test). This is in agreement with 
Table 1. Comparison of basic electrophysiological properties of hub neurons and LC interneurons. Measurements were obtained from whole-cell recordings in eight hub and eight LC interneurons (see SOM methods). $V_{\text {rest, }}$ resting membrane potential (corrected value; SOM); $R_{\text {input, input }}$ resistance; $V_{\text {threshold }}$ AP threshold (corrected value; SOM); AP width, AP width measured at halfmaximal amplitude. Asterisks indicate significant differences. $P<0.05$ was considered significant. The right column indicates the $P$ value given by Student or Mann-Whitney tests.

\begin{tabular}{lccc} 
Properties & Hub interneurons & LC interneurons & $P$ value \\
\hline$V_{\text {rest }}(\mathrm{mV})$ & $-66 \pm 5$ & $-64 \pm 5$ & 0.33 \\
$R_{\text {input }}(\mathrm{meg}$ ohms) & $379 \pm 85$ & $423 \pm 67$ & 0.69 \\
Capacitance $(\mathrm{pF})$ & $65 \pm 15$ & $54 \pm 15$ & 0.64 \\
$V_{\text {thresh }}(\mathrm{mV})$ & $-53 \pm 5^{*}$ & $-39 \pm 4$ & 0.04 \\
AP width (ms) & $2.05 \pm 0.5$ & $1.8 \pm 0.5$ & 0.74 \\
AP amplitude $(\mathrm{mV})$ & $45 \pm 6$ & $39 \pm 5$ & 0.43 \\
EPSP frequency $(\mathrm{Hz})$ & $4.5 \pm 1.5^{*}$ & $1.0 \pm 0.4$ & 0.03 \\
EPSP amplitude $(\mathrm{mV})$ & $2.1 \pm 0.3$ & $2.6 \pm 0.5$ & 0.51 \\
\hline
\end{tabular}

the imaging data and comparable to the highest synaptic connectivity rates reported for interneurons in the adult cortex (21). It therefore represents a high value given the fact that all the monosynaptically connected neurons were more than $100 \mu \mathrm{m}$ apart (the average distance between recorded neurons was $130 \pm 20 \mu \mathrm{m}, n=16$ pairs, fig. S5) and that the connection probability is very likely to increase with age $(30,31)$. We conclude that the functional connectivity of hub neurons is supported by an effective synaptic connectivity and propose that $\mathrm{HC}$ pyramidal neurons are more likely to operate within assemblies (32).

Because hub function may depend on differences in cellular excitability or synaptic strength $(33,34)$, we next examined the basic electrophysiological properties of hub neurons as compared to LC interneurons (Table 1). Of the basic features analyzed (SOM), hub neurons received more spontaneous excitatory postsynaptic potentials (EPSPs) and had a lower threshold for AP generation (Student's $t$ test, $P<0.05$ ). A lower AP threshold could indicate a more advanced maturation stage for hub neurons (35). Both properties should result in a more efficient activation of hub neurons by synaptic inputs.

Finally, because stimulation of hub neurons significantly affected the occurrence of GDPs, we examined their specific involvement in the spontaneous synchronization process. In agreement with previous estimates (36), the dynamic of a single GDP was characterized by a buildup of activity lasting on average $350 \mathrm{~ms}$ (Fig. 4, $n=$ 8 slices, SOM). Using cluster analysis (SOM), a stereotypical spatiotemporal synchronization pattern accounted for one-third of the GDPs within the recording period ( $33 \pm 2 \%, n=45$, Fig. 4). For each neuron, we estimated the average correlation and time of activation relative to all other cells in GDPs that clustered together (Fig. 4 and SOM). In almost half of the movies ( $n=20$ out of $45)$, the time correlation graph presented a bimodal distribution (Fig. 4B), indicating that GDPs repetitively started synchronizing neurons plotted on the left side of the distribution, whereas neurons on the right were activated last. By pooling the data from different slices $(n=7588$ neurons, 45 movies), we found that the majority of functional hubs clustered on the upper left region of the graph, indicating a more reliable activation at the onset of GDPs (Fig. 4B); this is in agreement with the lower AP threshold and higher synaptic drive described above. Other recorded neurons were evenly distributed across the correlation plot. Cell-attached and whole-cell recordings confirmed that cells activated at the buildup of synchronization indeed fired APs before the occurrence of GDPs ( $n=14$ neurons, fig. S1C). AP firing in hub neurons thus predicts network synchronization in the developing CA3 region.

This study shows that a scale-free topology can underlie synchronous network patterns in living cortical networks. We suggest that hub neurons, composed of a subpopulation of GABAergic interneurons, orchestrate spontaneous network synchronization. Two different morphological types of hub neurons could be distinguished within our sample data set: (i) cells displaying a long axon spanning regions with sparse collaterals, and (ii) basketlike neurons with a dense but more local arborization pattern. Network synchronization could be triggered by phasic stimulation only in basketlike hub neurons (Fig. 3A and fig. S5). In the adult hippocampus, long-range projecting GABAergic hippocampal interneurons have been described (37) and their hub function has been suggested but never been probed (4). Perhaps the long-axon hub neurons act as connector hubs, whereas basketlike hubs have a local hub function (19). Regardless, the present results confirm the crucial role of GABAergic transmission in shaping network patterns at early developmental stages, when GABA exerts a complex excitation/ shunting inhibition action $(38,39)$. The spontaneous activation, before synchrony, of hub neurons with many direct postsynaptic connections is compatible with excitatory actions of GABA. However, hub cell stimulation also often slowed down network oscillations and in some extreme cases completely desynchronized activity. One possible explanation is that the shunting actions of GABA retard or prevent synchronization. However, other possibilities cannot be excluded, including a phase-resetting effect by which a hub cell can either advance or delay bursting in intrinsically oscillating neurons (39), depending on their phase at the time of the hub input (40).

Single neurons can trigger population synchronization in the disinhibited adult CA3 region (41) or elicit a chain of cell activation in the cortex that can translate into behavior or switch the global brain state (42-44). Therefore, the demonstration that hub neurons functionally operate in the brain helps bridge the gap between single-cell and network activity. This finding should facilitate the investigation of the mechanisms by which many physiological and pathological network oscillations are generated.

References and Notes

1. G. Buzsáki, Rhythms of the Brain (Oxford Univ. Press, Oxford, 2006).

2. L. C. Katz, C. J. Shatz, Science 274, 1133 (1996).

3. Y. Ben Ari, Trends Neurosci. 24, 353 (2001)

4. G. Buzsaki, C. Geisler, D. A. Henze, X. J. Wang, Trends Neurosci. 27, 186 (2004).

5. G. Grinstein, R. Linsker, Proc. Natl. Acad. Sci. U.S.A. 102, 9948 (2005)

6. R. J. Morgan, I. Soltesz, Proc. Natl. Acad. Sci. U.S.A. 105, 6179 (2008).

7. D. J. Watts, S. H. Strogatz, Nature 393, 440 (1998)

8. A. L. Barabasi, R. Albert, Science 286, 509 (1999).

9. S. Boccaletti, V. Latora, Y. Moreno, M. Chavez, D.-U. Hwang, Phys. Rep. 424, 175 (2006).

10. L. A. Amaral, A. Scala, M. Barthelemy, H. E. Stanley, Proc. Natl. Acad. Sci. U.S.A. 97, 11149 (2000).

11. R. L. Buckner et al., J. Neurosci. 29, 1860 (2009).

12. V. M. Eguiluz, D. R. Chialvo, G. A. Cecchi, M. Baliki, A. V. Apkarian, Phys. Rev. Lett. 94, 018102 (2005).

13. D. Eytan, S. Marom, J. Neurosci. 26, 8465 (2006).

14. O. Sporns, C. J. Honey, R. Kotter, PLoS One 2, e1049 (2007).

15. D. J. de Solla Price, Science 149, 510 (1965).

16. E. V. Lubenov, A. G. Siapas, Nature 459, 534 (2009).

17. K. V. Srinivas, R. Jain, S. Saurav, S. K. Sikdar, Eur. J. Neurosci. 25, 3276 (2007)

18. S. Yu, D. Huang, W. Singer, D. Nikolic, Cereb. Cortex 18, 2891 (2008)

19. E. Bullmore, O. Sporns, Nat. Rev. Neurosci. 10, 186 (2009).

20. S. Song, P. J. Sjostrom, M. Reigl, S. Nelson, D. B. Chklovskii, PLoS Biol. 3, e68 (2005).

21. A. M. Thomson, C. Lamy, Front. Neurosci. 1, 19 (2007).

22. N. C. Spitzer, Nature 444, 707 (2006).

23. A. A. Cattani, V. D. Bonfardin, A. Represa, Y. Ben-Ari, L. Aniksztejn, J. Neurophysiol. 98, 2324 (2007).

24. M. Canepari, F. Mammano, S. G. Kachalsky, R. Rahamimoff, E. Cherubini, Cell Calcium 27, 25 (2000).

25. L. Menendez de la Prida, S. Bolea, ]. V. Sanchez-Andres, Eur. J. Neurosci. 10, 899 (1998).

26. V. Crepel et al., Neuron 54, 105 (2007).

27. S. Bolea, J. V. Sanchez-Andres, X. Huang, J. Y. Wu, J. Neurophysiol. 95, 552 (2006).

28. T. F. Freund, G. Buzsáki, Hippocampus 6, 347 (1996)

29. N. Tamamaki et al., J. Comp. Neurol. 467, 60 (2003).

30. D. Doischer et al., J. Neurosci. 28, 12956 (2008).

31. L. Groc, B. Gustafsson, E. Hanse, Eur. J. Neurosci. 17, 1873 (2003)

32. K. D. Harris, J. Csicsvari, H. Hirase, G. Dragoi, G. Buzsaki, Nature 424, 552 (2003).

33. L. Wittner, R. Miles, J. Physiol. 584, 867 (2007).

34. F. Strata et al., J. Neurosci. 17, 1435 (1997).

35. S. Rheims et al., J. Neurophysiol. 100, 609 (2008)

36. L. M. Prida, ]. V. Sanchez-Andres, J. Neurophysiol. 82 , 202 (1999)

37. S. Jinno et al., J. Neurosci. 27, 8790 (2007).

38. Y. Ben-Ari, ]. L. Gaiarsa, R. Tyzio, R. Khazipov, Physiol. Rev. 87, 1215 (2007).

39. S. T. Sipila, K. Huttu, I. Soltesz, J. Voipio, K. Kaila, J. Neurosci. 25, 5280 (2005).

40. H. Y. Jeong, B. Gutkin, Neural Comput. 19, 706 (2007).

41. L. M. de la Prida, G. Huberfeld, I. Cohen, R. Miles, Neuron 49, 131 (2006). 
42. M. Brecht, M. Schneider, B. Sakmann, T. W. Margrie, Nature 427, 704 (2004).

43. G. Molnar et al., PLoS Biol. 6, e222 (2008).

44. C. Y. Li, M. M. Poo, Y. Dan, Science 324, 643 (2009).

45. We thank D. Aronov, M. Colonnese, ]. Epsztein, B. Fernandez, G. Fishell, C. Holmgren, B. Gutkin, M. Milh, and R. Khazipov for helpful suggestions and critical comments; C. Allene, K. Bennouar, and F. Michel for help with the experiments; and K. Obata and K. Vogt for kindly providing GAD67-EGFP Ki mice. This work was supported by grants from INSERM, the Ville de Marseille and Region Provence Alpes Côte d'Azur, the Fondation pour la Recherche Médicale, the Agence Nationale pour la Recherche, the Fondation pour la Recherche sur le Cerveau, and the Fondation Bettencourt Schueller. R.C. and A.R. were funded by the CNRS. M. Goldin and P. Bonifazi were funded by Framework Program 6 (FP6) and FP7-Intra-European Fellowships for career development.
Supporting Online Material

www.sciencemag.org/cgi/content/full/326/5958/1419/DC1

Materials and Methods

Figs. S1 to S5

References

Movie S1

27 April 2009; accepted 23 September 2009

$10.1126 /$ science. 1175509

\title{
Deletion of Atoh1 Disrupts Sonic Hedgehog Signaling in the Developing Cerebellum and Prevents Medulloblastoma
}

\author{
Adriano Flora, ${ }^{1}$ Tiemo ]. Klisch, ${ }^{1,2}$ Gabriele Schuster, ${ }^{1}$ Huda Y. Zoghbi ${ }^{1,2,3,4 *}$ \\ Granule neuron precursors (GNPs) are the most actively proliferating cells in the postnatal \\ nervous system, and mutations in pathways that control the GNP cell cycle can result in \\ medulloblastoma. The transcription factor Atoh1 has been suspected to contribute to GNP \\ proliferation, but its role in normal and neoplastic postnatal cerebellar development remains \\ unexplored. We show that Atoh1 regulates the signal transduction pathway of Sonic Hedgehog, \\ an extracellular factor that is essential for GNP proliferation, and demonstrate that deletion of \\ Atoh1 prevents cerebellar neoplasia in a mouse model of medulloblastoma. Our data shed light on \\ the function of Atoh1 in postnatal cerebellar development and identify a new mechanism \\ that can be targeted to regulate medulloblastoma formation.
}

$\mathrm{D}$ isruption of the delicate balance between proliferation and differentiation in cerebellar granule neuron precursors (GNPs) underlies medulloblastoma, the most common pediatric tumor of the nervous system $(1,2)$. A class of particularly aggressive medulloblastomas associated with very poor prognosis show high expression of Atoh1 (3), a transcription factor highly expressed in GNPs also known as Math1 (4), and recent in vitro studies proposed that Atoh 1 might be involved in neoplastic proliferation $(5,6)$. Given that deletion of Atohl in mice results in perinatal death (7), the function of this transcription factor in the developing postnatal cerebellum has remained opaque.

To delete Atoh1 in the postnatal developing cerebellum, we crossed $A$ toh $1^{\text {flox/flox }}$ mice $(8)$ with mice carrying the gene coding for a tamoxifeninducible Cre recombinase in the Rosa locus (R26CreER) (9) and a null allele of Atohl $(10,11)$. After activation of Cre by tamoxifen, Rosa ${ }^{\text {CreER }}$;Atoh $1^{+/ \text {flox }}$ animals (designated here as Atoh $1^{\text {wt }}$ ) maintain one functional allele of Atoh1, whereas Rosa ${ }^{\text {CreER }}$;Atoh $1^{-/ \text {flox }}$ mice (designated here as $A t o h 1^{\Delta}$ ) lose Atoh1 expression. We injected postnatal day 3 (P3) animals and analyzed

${ }^{1}$ Department of Molecular and Human Genetics, Baylor College of Medicine, Houston, TX 77030, USA. ${ }^{2}$ Howard Hughes Medical Institute, Baylor College of Medicine, Houston, TX 77030, USA. ${ }^{3}$ Departments of Neuroscience and Pediatrics, Baylor College of Medicine, Houston, TX 77030, USA. ${ }^{4}$ Program in Developmental Biology, Baylor College of Medicine, Houston, TX 77030, USA

*To whom correspondence should be addressed. E-mail: hzoghbi@bcm.edu their cerebella 3 days later. Nissl staining of matching sections of the external granule layer (EGL), the neuroepithelium formed by GNPs,
A

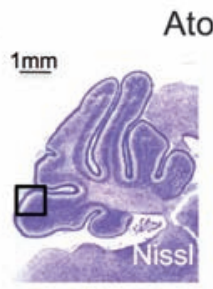

Atoh 1 wt

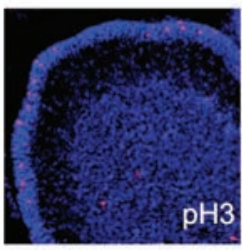

C

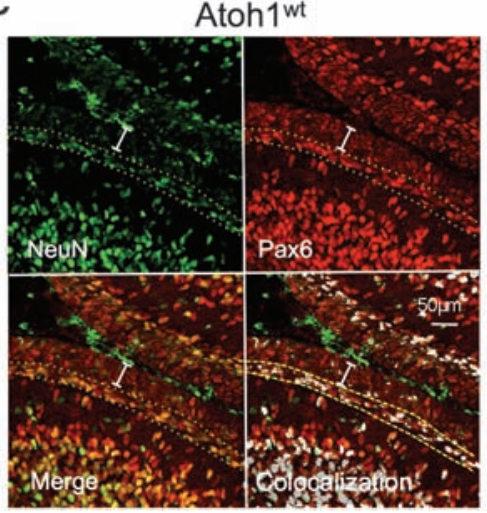

revealed that $A t o h 1^{\Delta}$ animals had a much thinner EGL than that of their $A t o h I^{\text {wt }}$ littermates (fig. S1). Using phospohistone $\mathrm{H} 3$ staining to visualize the $M$ phase of the cell cycle and Tuj1 for neural differentiation, we found that the EGL of Atoh ${ }^{\Delta}$ mice had been depleted of cycling immature precursors (Fig. 1, A and B, and fig. S2). Staining for active caspase 3 did not reveal any apoptosis in the EGL of Atoh $1^{\Delta}$ mice (fig. S1, E and F). We thus investigated whether deletion of Atoh1 triggers the GNP differentiation to granule neurons or induces these cells to transdifferentiate to other cell types. Shown in fig. S2, cells still populating the surface of Atoh $1^{\Delta}$ cerebellum that had deleted Atoh1 still expressed Zic1, a marker of differentiating postmitotic EGL cells and mature granule neurons, making transdifferentiation unlikely. Proliferating GNPs express Pax6 at low levels, whereas differentiating postmitotic precursors show high expression of Pax6 and turn on the neural differentiation marker NeuN. The cells residing on the surface of the cerebellum of Atoh $^{\Delta}$ expressed high levels of

B

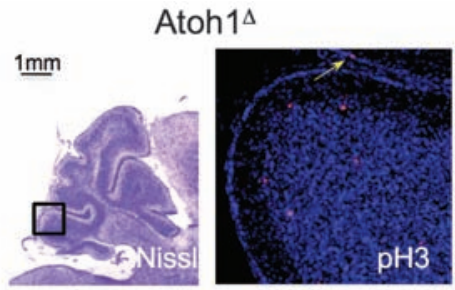

D

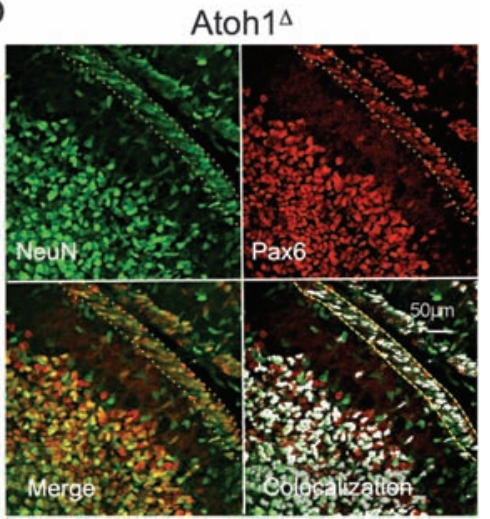

Fig. 1. Atoh1 deletion disrupts GNP proliferation and induces differentiation. (A and B) Phosphohistone H3 staining of cerebella of animals injected with tamoxifen. (Left) Nissl staining of cerebella of animals injected with tamoxifen. The boxed regions represent the images to their right, showing the staining for phosphohistone H3. The arrow in (B) indicates a single cycling cell in the Atoh ${ }^{\Delta}$ EGL. (C and D) Pax6 and NeuN staining shows the immature GNPs [(C), white bar] not expressing NeuN and the differentiating population [(C) and (D), between the yellow dotted lines] coexpressing both markers. The colocalization pattern is shown on the bottom right. 